\title{
The role of thermal radiation on the boundary layer past a stationary flat plate with constant surface boundary condition
}

\author{
Basant K Jha ${ }^{1}$, Gabriel Samaila ${ }^{* 2}$ \\ ${ }^{1}$ Department of Mathematics, Ahmadu Bello University, Zaria, Nigeria \\ 2Department of Mathematics, Air Force Institute of Technology, Kaduna, Nigeria
}

Keywords

Thermal radiation, boundary layer, constant surface boundary condition and flat plate.

\section{Abstract}

This study is devoted to investigating the importance of thermal radiation on the boundary layer over a horizontal surface considering classical constant surface boundary condition. The mathematical model consists of coupled two-dimensional partial differential equations which are transformed to the set of ordinary differential equations via the similarity transformation. The final set of dimensionless equations is solved numerically using Runge Kutta Fehlberg (RKF45) method in Maple software. The significant effect of the thermal radiation is examined using four fluids namely; water, Sulphur oxide, air and mercury whose respective Prandtl numbers are $7,2,0.72$ and 0.044 . The influence of other prominent parameters affecting the flow formation and temperature profile is demonstrated using tables and graphs. The results indicated that the thermal boundary layer thickness could be increase by reducing the Prandtl number. The results also showed that increasing the thermal radiation parameter has a positive impact on the boundary layer thickness. The heat transfer rate could be improved by increasing thermal radiation or decreased by increasing the values of the Prandtl number. Regarding the temperature gradient, an observable increasing is seen far from the flat plate with the growing of thermal radiation whereas the opposite trend is true near the plate surface.

\section{Introduction}

Recently, analysis of boundary layer flow has witnessed global attention because of its various applications in the areas such as separation and attachment in aerodynamics, golf ball aerodynamics, mixing enhancement, heat transfer enhancement etc. Early investigations such as[1]-[6] explored the behaviour of fluid flow on the boundary layer past a vertical/horizontal wall. Furthermore, recent work such as [7]-[10] presented the influence of the active parameters on boundary layer flow considering various geometries. Similarity transformation is an important instrument used to transform PDE to ODE. It is mostly used in boundary layer analysis due to the coupled and highly nonlinear nature of the boundary layer model. Aziz (2009) [11] present a similarity solution for boundary layer flow with emphasis on the convective boundary condition. Makinde and Olanrewaju (2010) [12] also adopted a similarity solution to study boundary layer flow with the buoyancy effect. Some literature on similarity solution can be found in [13]-[17].

In the last decade, the roles of thermal radiation has attracted the attention of several workers due to its application in industries. Thermal radiation is applied in the design of industrial equipment such as gas turbines, nuclear power plants and missiles. Raju et al.(2013)[18] explored the implication of the heat source and thermal radiation on MHD natural convection over a vertical wall. Sharma et al.(2012) [19] and Thakur et al (2014) [20] presented the influence of Dufour and Soret on MHD mixed convection flow over a vertical plate. Hayat et al. (2013) [21] also presented the importance of thermal radiation on MHD 3-D flow.

An oscillatory flow of natural convection with thermal radiation over a vertical wall was studied by El-Hakiem (2000) [22]. Makinde (2008) [23] also presented boundary layer flow with mass transfer and thermal radiation past a continuously moving plate. Jha et al.(2016) [24] presented the importance of suction/injection on MHD natural convection flow with thermal radiation. Hossain et al.[25] addressed the radiation effect on mixed convection over a vertical wall. Raptis
(1998)[26] published work on free convection flow through a porous medium with radiation. Makinde (2005)[27] expounded on the effects of mass transfer and thermal radiation on natural convection past a continuously moving plate. Ibrahim [28] presented a combined influence of absorption radiation on unsteady MHD free convection flow over a vertically continuous moving wall. Suneetha et al.(2008)[29] investigated the effect of thermal radiation on MHD natural convection flow over a vertical wall considering the variable concentration and temperature.

To the best of the authors' knowledge, the effect of thermal radiation on the boundary layer flow over a horizontal plate using constant surface boundary condition has been neglected by several researchers. In our contribution to the existed literature on boundary layer, we demonstrated the significant role of the thermal radiation over a horizontal surface using constant surface boundary condition. As it customarily in boundary layer analysis, $\infty$ is replaced by 10 in all our calculation.

\section{Mathematical Formulation}

Consider hydrodynamics and the boundary layer flow with thermal radiation effect past a horizontal plate. Let $U_{\infty}$ be the uniform velocity of the fluid moving over the plate's surface at temperature $T_{\infty}$. The $x$-axis is considered to be along with the plate while the y-axis normal to the plate. Following Aziz(2009) [11], the governing can be formulated as

$\frac{\partial u}{\partial x}+\frac{\partial v}{d y}=0$

$u \frac{\partial u}{\partial x}+v \frac{\partial u}{d y}=v \frac{\partial^{2} u}{\partial y^{2}}$

$u \frac{\partial T}{\partial x}+v \frac{\partial T}{d y}=\alpha\left[\frac{\partial^{2} T}{\partial y^{2}}-\frac{1}{K} \frac{\partial q_{r}}{\partial y}\right]$
*Corresponding Author: gabbbooooo@yahoo.com

G. Samaila ORCID https://orcid.org/0000-0002-7463-9427
Received 20 Jan 2021 Revised 06 Feb 2021 Accepted 12 Feb 2021 Journal of Nature, Science \& Technology 2 (2021) 7-11 
Where $u$ and $v$ are respectively velocity components along and normal to the plate and $K$ denotes the thermal conductivity. The quantity $q_{r}$ in Eqn. (3) denotes the radiative heat flux normal to the plate and it can be simplified through Rosseland diffusion approximation as.

$q_{r}=-\frac{4 \sigma \partial T^{2}}{3 k^{*} \partial y}$

The boundary conditions for the velocity are as follows;

$U(x, 0)=v(x, 0)=0$

$u(x, \infty)=U_{\infty}$

The plate surface is considered to be heating through convection by the hot fluid at the temperature $T_{f}$ with heat flux coefficient $T_{f}$. Thus, the temperature boundary condition can be written as

$T(x, y)=T_{0}$

$T(x, \infty)=T_{\infty}$

We define the similarity variable $\eta$, stream function $f(\eta)$ and the dimensionless parameters defined as

$\eta=y \sqrt{\frac{U_{\infty}}{v x},} \quad u=U_{\infty} f^{\prime}(\eta)$,

$v=\frac{1}{2} \sqrt{\frac{U_{\infty} v}{x}}\left(\eta f^{\prime}(\eta)-f\right)$

$R=\frac{4 \sigma\left(T_{f}-T_{\infty}\right)^{3}}{k^{*} K}, \quad C_{T}=\frac{T_{\infty}}{T_{f}-T_{\infty}}$

$\operatorname{Pr}=\frac{\mu C_{T}}{K}, \quad \theta=\frac{\left(T-T_{\infty}\right)}{\left(T_{f}-T_{\infty}\right)}$.

The reduced dimensionless equations and boundary conditions can be written as

$2 f(\eta)^{\prime \prime \prime}(\eta)+f(\eta) f(\eta)^{\prime \prime}(\eta)=0$

$\theta^{\prime}(\eta)\left[1+\frac{4 R}{3}\left(\theta+C_{T}\right)^{2}\right]+4 R\left[C_{T}+\theta\right]^{2}\left[\theta^{\prime}(0)\right]^{2}+$

$\frac{1}{2} \operatorname{Pr} \theta^{\prime}(\eta) f(\eta)=0$

$$
\begin{aligned}
& f(0)=f^{\prime}(0)=0, \quad f^{\prime}(\infty)=1 \\
& \theta(0)=1, \quad \theta(\infty)=0
\end{aligned}
$$

Where prime represent derivative with respect to $\eta$ and all the parameters in the above equations are defined in the nomenclature section.

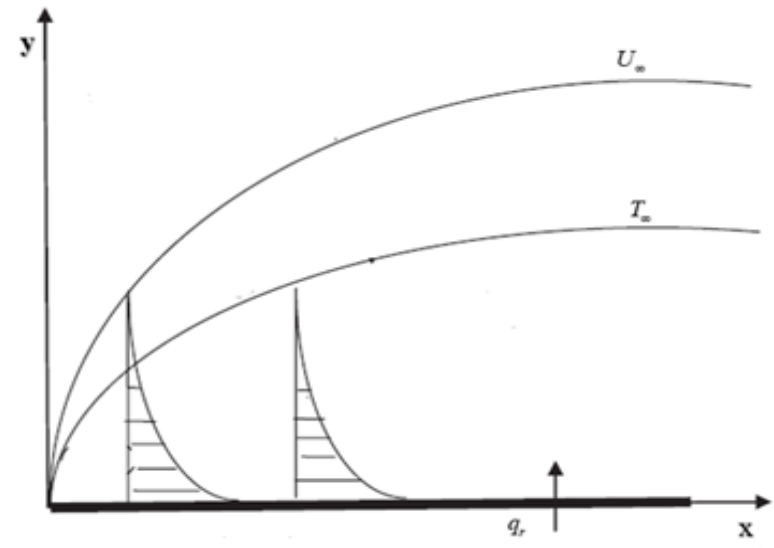

Figure 1: Physical model

\section{Results and Discussion}

In this section, the influence of embedded parameters such as temperature difference $\left(C_{T}\right)$, Prandtl number $(P r)$ and the thermal radiation $(R)$ on the plate temperature and the flow formation are fully discussed in details. The numerical solution to the governing equation is obtained through the RKF45 method in maple software. It is found that assigning the value of $\infty \geq 10$ gives excellent results compared to $\infty<10$ thus, $\infty=10$ is used throughout the simulation. The study considered four working fluids namely; water $(\operatorname{Pr}=7)$, Sulphur oxide $(P r=2)$, air $(P r=0.72)$ and mercury $(P r=0.044)$. Figure 2-5 portray the importance of varying $R$ on the plate temperature for the four fluids under examination. It is observed from the figures that the temperature profiles for the four fluids could be enhanced by increasing the thermal radiation effect. Regarding the temperature gradient, an observable increasing is seen far from the flat plate with the growing of thermal radiation whereas the opposite trend is true near the plate surface as depicted in Figure 6. The role of $C_{T}$ on the temperature distribution is demonstrated in Figure 7. The figure revealed that increasing the temperature difference between the ambient and free stream leads to an increase in the temperature distribution. The implication of $P r$ is portrayed in Figure 8 where $P r$ augment has the potential to reduce the thickness of the boundary layer. Generally, the momentum and thermal diffusion or the velocity and thermal boundary layer coincide when $\operatorname{Pr}=1$. However, for liquid fluid $\operatorname{Pr}>1$, the viscous/momentum diffusivity dominate the fluid flow and in turn, the velocity boundary layer becomes thicker than the thermal boundary layer whereas contrary behaviour is true for $\operatorname{Pr}<1$. This can be seen in Figure 7 where the temperature profile of water reached its lowest values at approximately half $\left(\frac{1}{2}\right)$ of the sulphur oxide, one-third $\left(\frac{1}{3}\right)$ of air and one-fifth $\left(\frac{1}{5}\right)$ of mercury.

Table 1 represents the impact of $\mathrm{R}$ on the Nusselt number for various fluid under examination with $C_{T}=0.2$. It is worthy to remark from the table that the Nusselt number drops as the values of $R$ increase whereas increases with growing values of Pr. Therefore, the heat transfer rate could be improved by increasing $\mathrm{R}$ or decreased by increasing the values of $\mathrm{Pr}$.

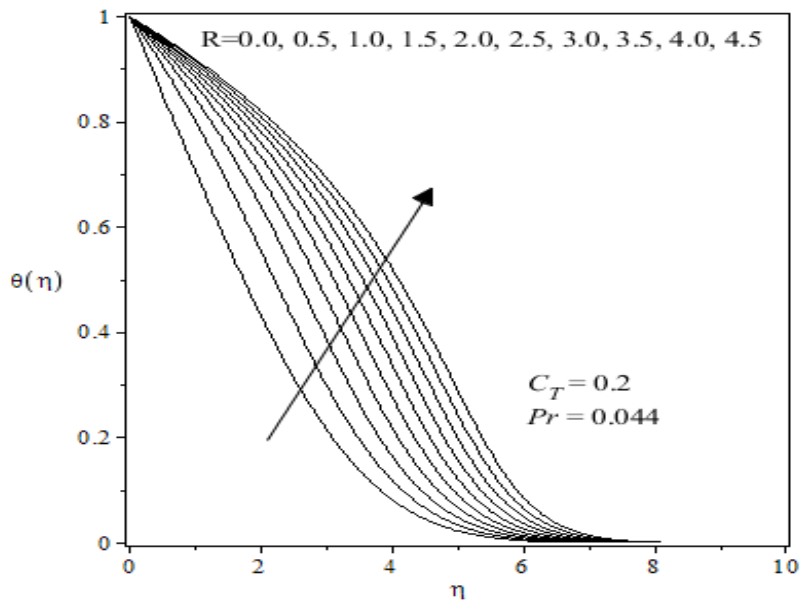

Figure 2: Variation of $R$ on the temperature profile. 


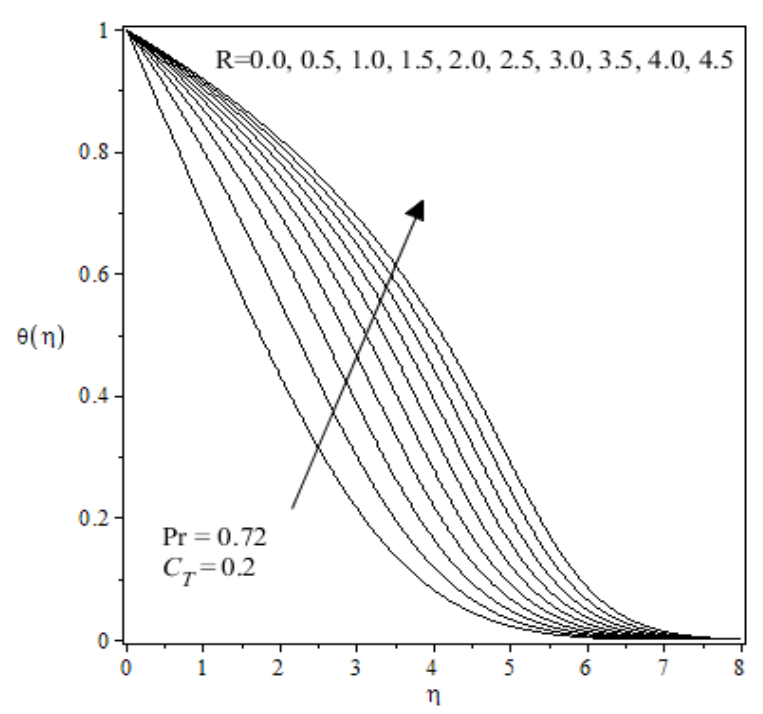

Figure 3: Variation of $R$ on the temperature profile.

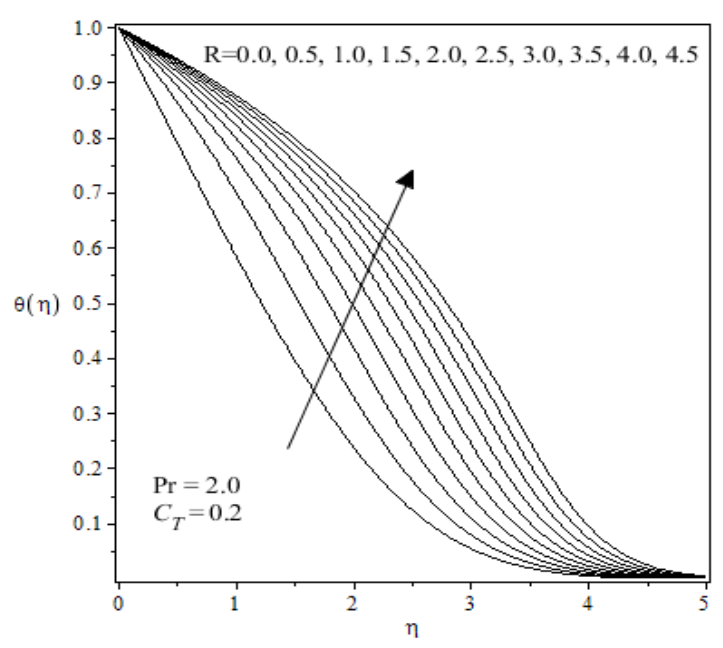

Figure 4: Variation of $R$ on the temperature profile

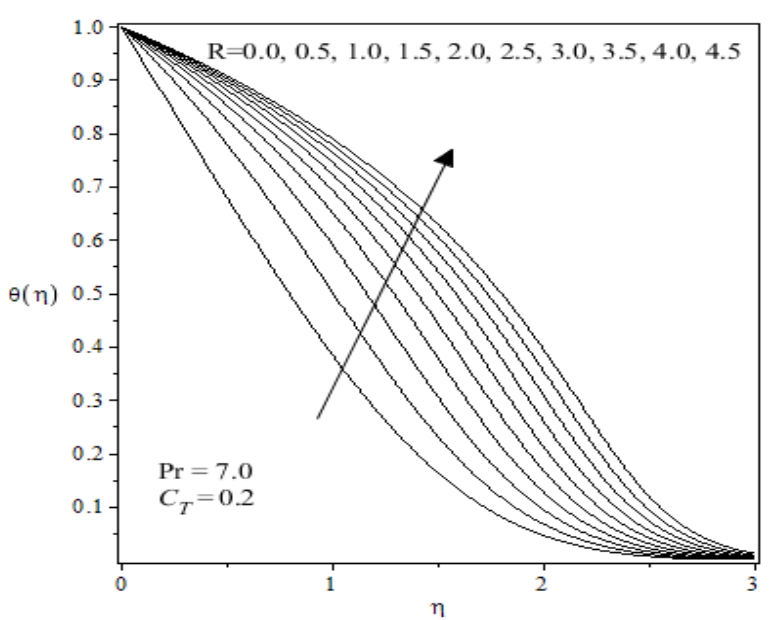

Figure 5: Variation of $R$ on the temperature profile.

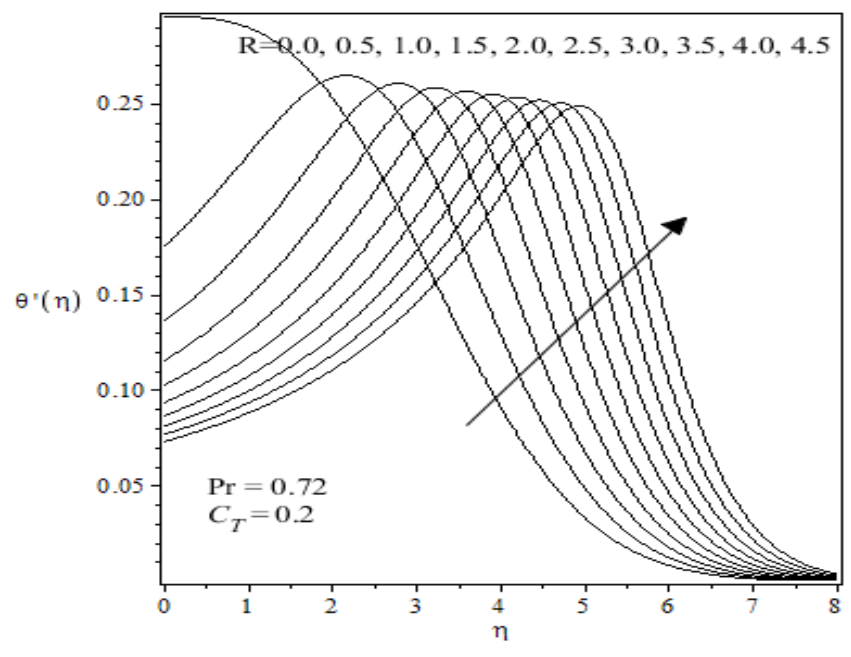

Figure 6: Variation of $R$ on the temperature gradient profile.

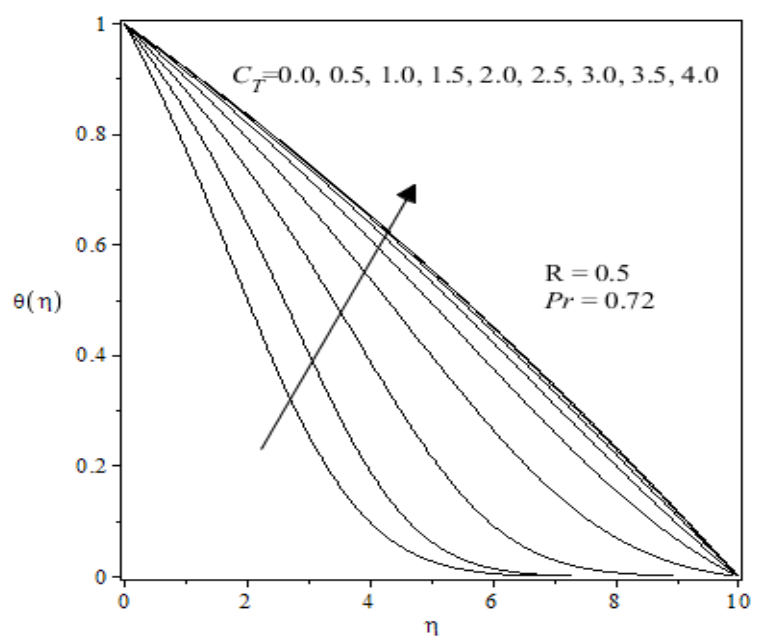

Figure 7: Variation of $C_{T}$ on the temperature profile

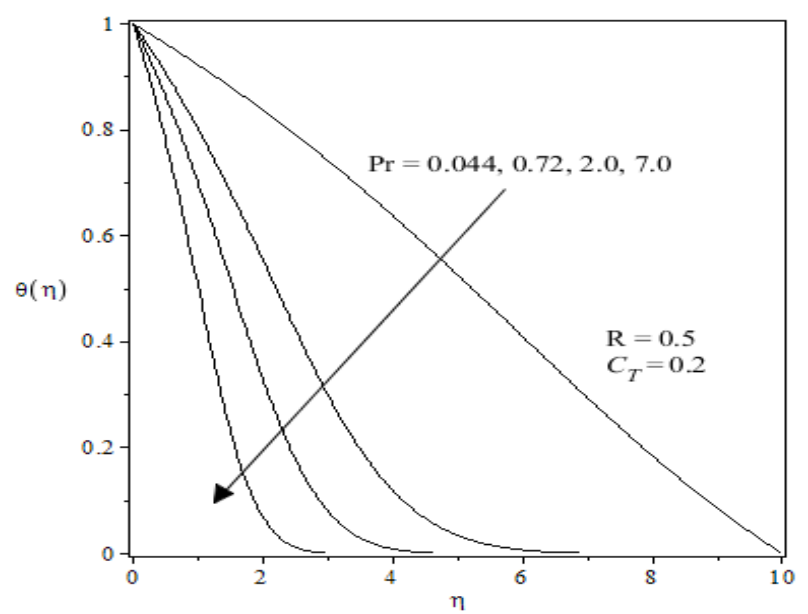

Figure 8: Variation of $\operatorname{Pr}$ on the thermal boundary layer. 
Table 1. Numerical values for $-\theta^{\prime}(0)$ for $C_{T}=0.2$

\begin{tabular}{ccccc}
\hline$R$ & $P r=0.044$ & $\operatorname{Pr}=0.72$ & $\operatorname{Pr}=2.0$ & $\operatorname{Pr}=7.0$ \\
\hline 0.0 & 0.12166 & 0.29564 & 0.42231 & 0.64592 \\
0.5 & 0.07355 & 0.17572 & 0.25160 & 0.38525 \\
1.0 & 0.05848 & 0.13650 & 0.19598 & 0.30047 \\
1.5 & 0.05105 & 0.11605 & 0.16708 & 0.25651 \\
2.0 & 0.04664 & 0.10309 & 0.14884 & 0.22884 \\
2.5 & 0.04372 & 0.09396 & 0.13603 & 0.20944 \\
3.0 & 0.04166 & 0.08708 & 0.12640 & 0.19488 \\
3.5 & 0.04012 & 0.08164 & 0.11881 & 0.18343 \\
4.0 & 0.03893 & 0.07719 & 0.11262 & 0.17412 \\
& & & & \\
\hline
\end{tabular}

\section{Conclusion}

The importance of thermal radiation on the boundary layer flow over a stationary flat plate is examined. The numerical solution to the sets of the differential equation is obtained using the RKF45 method. The significant effect of the thermal radiation and other active parameters are examined using four fluids namely; water, Sulphur oxide, air and mercury. The results showed that;

(i). The thickness of the boundary layer decreases with growing values of $\mathrm{Pr}$.

(ii). Augmenting $\mathrm{R}$ has a positive impact on the temperature distribution for the four fluids under examination.

(iii). The temperature difference augment has an increasing impact on the plate temperature for the four fluids.

(iv). The heat transfer rate could be improved by increasing $\mathrm{R}$ or decreased by increasing the values of $\mathrm{Pr}$.

\section{Nomenclature}

$R$ Thermal radiation (dimensionless)

$q_{r} \quad$ Radiative heat flux

$\mathrm{Pr}$ Prandtl number (dimensionless)

$\theta \quad$ Temperature (dimensionless)

$T$ Temperature

Re Reynold number

$v \quad$ Kinematic viscosity

$k^{*} \quad$ mean absorption

$C_{T} \quad$ Temperature difference (dimensionless)

$u, v$ velocity components

$\boldsymbol{\sigma}$ Stefan Boltzmann constant

\section{Declaration of Conflict of Interests}

The authors declare that there is no conflict of interest. They have no known competing financial interests or personal relationships that could have appeared to influence the work reported in this paper.

\section{References}

[1.] E. A. Koldenhof, "Laminar boundary layers on continuous flat and cylindrical surfaces," AIChE J., vol. 9, no. 3, pp. 411-418, 1963.

[2.] H. Blasius, "The boundary layers in fluids with little friction," 1950.

[3.] M. D. A. Sattar and H. Kalim, "Unsteady free-convection interaction with thermal radiation in a boundary layer flow past a vertical porous plate," J. Math. Phys. Sci, vol. 30, no. 1, pp. 2537, 1996.

[4.] M. Taghite, B. Barake, A. Rahmattulla, and H. LanchonDucauquis, "Evaluation of the thermal boundary layer in the plate of a heat exchanger and the error estimate," Comput. Methods Appl. Mech. Eng., vol. 178, no. 1, pp. 141-152, 1999.

[5.] P. Neofytou, A. G. Venetsanos, D. Vlachogiannis, J. G. Bartzis, and A. Scaperdas, "CFD simulations of the wind environment around an airport terminal building," Environ. Model. Softw., vol. 21, no. 4, pp. 520-524, 2006.

[6.] M. D. Aljoufi and A. Ebaid, "Effect of a convective boundary condition on boundary layer slip flow and heat transfer over a stretching sheet in view of the exact solution," J. Theor. Appl. Mech., vol. 46, no. 4, pp. 85-95, 2016.

[7.] B. K. Jha and G. Samaila, "Thermal radiation effect on boundary layer over a flat plate having convective surface boundary condition," SN Appl. Sci., vol. 2, no. 3, p. 381, 2020.

[8.] G. Oh, K. M. Noh, H. Park, and J.-I. Choi, "Extended synthetic eddy method to generate inflow data for turbulent thermal boundary layer," Int. J. Heat Mass Transf., vol. 134, pp. 1261-1267, 2019.

[9.] I. V Egorov, N. V Palchekovskaya, A. O. Obraz, and A. V Fedorov, "Effects of injection on heat transfer and the boundary-layer instability for a hypersonic blunt body configuration," Int. J. Heat Mass Transf., vol. 149, p. 119197, 2020.

[10.] B. J. Gireesha, M. Umeshaiah, B. C. Prasannakumara, N. S. Shashikumar, and M. Archana, "Impact of nonlinear thermal radiation on magnetohydrodynamic three dimensional boundary layer flow of Jeffrey nanofluid over a nonlinearly permeable stretching sheet," Phys. A Stat. Mech. its Appl., p. 124051, 2020.

[11.] A. Aziz, "A similarity solution for laminar thermal boundary layer over a flat plate with a convective surface boundary condition," Commun. Nonlinear Sci. Numer. Simul., vol. 14, no. 4, pp. 10641068, 2009.

[12.] O. D. Makinde and P. O. Olanrewaju, "Buoyancy effects on thermal boundary layer over a vertical plate with a convective surface boundary condition," J. Fluids Eng., vol. 132, no. 4, p. 44502, 2010.

[13.] S. Yao, T. Fang, and Y. Zhong, "Heat transfer of a generalized stretching/shrinking wall problem with convective boundary conditions," Commun. Nonlinear Sci. Numer. Simul., vol. 16, no. 2, pp. 752-760, 2011.

[14.] P. D. Weidman, D. G. Kubitschek, and A. M. J. Davis, "The effect of transpiration on self-similar boundary layer flow over moving surfaces," Int. J. Eng. Sci., vol. 44, no. 11, pp. 730-737, 2006.

[15.] W. Ibrahim and O. D. Makinde, "The effect of double stratification on boundary-layer flow and heat transfer of nanofluid over a vertical plate," Comput. Fluids, vol. 86, pp. 433-441, 2013.

[16.] S. Mukhopadhyay, K. Bhattacharyya, and G. C. Layek, "Steady boundary layer flow and heat transfer over a porous moving plate in presence of thermal radiation," Int. J. Heat Mass Transf., vol. 54, no. 13, pp. 2751-2757, 2011.

[17.] B. K. Jha and G. Samaila, "A Similarity Solution for Natural Convection Flow near a Vertical Plate with Thermal Radiation," Microgravity Sci. Technol., pp. 1-8, 2020.

[18.] R. S. Raju, K. Sudhakar, and M. Rangamma, "The effects of thermal radiation and Heat source on an unsteady MHD free convection flow past an infinite vertical plate with thermal diffusion and diffusion thermo," J. Inst. Eng. Ser. C, vol. 94, no. 2, pp. 175-186, 2013.

[19.] B. K. Sharma, K. Yadav, N. K. Mishra, and R. C. Chaudhary, "Soret and Dufour effects on unsteady MHD mixed convection flow past a radiative vertical porous plate embedded in a porous medium with chemical reaction," Appl. Math., vol. 3, no. 7, p. 717, 2012.

[20.] M. Thakur, M. V. Krishna, and others, "• the effects of soret and dufour on an unsteady mhd free convection flow past an infinite vertical plate with thermal radiation and heat source," Int. $J$. Math. Arch. EISSN 2229-5046, vol. 5, no. 5, 2014.

[21.] T. Hayat, M. Awais, and S. Asghar, "Radiative effects in a threedimensional flow of MHD Eyring-Powell fluid," J. Egypt. Math. Soc., vol. 21, no. 3, pp. 379-384, Oct. 2013. 
[22.] M. A. El-Hakiem, "MHD oscillatory flow on free convection-radiation through a porous medium with constant suction velocity," J. Magn. Magn. Mater., vol. 220, no. 2-3, pp. 271-276, 2000.

[23.] O. D. Makinde and A. Ogulu, "The Effect Of Thermal Radiation On The Heat And Mass Transfer Flow Of A Variable Viscosity Flund Past A Vertıcal Porous Plate Permeated By A Transverse Magnetıc Field," Chem. Eng. Commun., vol. 195, no. 12, pp. 1575-1584, Aug. 2008.

[24.] M. M. Bhatti and M. M. Rashidi, "Effects of thermo-diffusion and thermal radiation on Williamson nanofluid over a porous shrinking/stretching sheet," J. Mol. Liq., vol. 221, pp. 567-573, Sep. 2016.

[25.] M. A. Hossain and H. S. Takhar, "Radiation effect on mixed convection along a vertical plate with uniform surface temperature," Heat Mass Transf., vol. 31, no. 4, pp. 243-248, 1996.

[26.] A. Raptis, "Radiation and free convection flow through a porous medium," Int. Commun. Heat Mass Transf., vol. 25, no. 2, pp. 289295, 1998.

[27.] O. D. Makinde, "Free convection flow with thermal radiation and mass transfer past a moving vertical porous plate," Int. Commun. Heat Mass Transf., vol. 32, no. 10, pp. 1411-1419, 2005.

[28.] F. S. Ibrahim, A. M. Elaiw, and A. A. Bakr, "Effect of the chemical reaction and radiation absorption on the unsteady MHD free convection flow past a semi infinite vertical permeable moving plate with heat source and suction," Commun. Nonlinear Sci. Numer. Simul., vol. 13, no. 6, pp. 1056-1066, 2008.

[29.] S. Suneetha, N. Bhaskar Reddy, and V. Ramachandra Prasad, "Thermal radiation effects on MHD free convection flow past an impulsively started vertical plate with variable surface temperature and concentration," J. Nav. Arch. Mar. Eng, vol. 5, no. 2, pp. 57-70, 2008.

\section{How to Cite This Article}

Basant $\mathrm{K}$ Jha, Gabriel Samaila, The role of thermal radiation on the boundary layer past a stationary flat plate with constant surface boundary condition, Journal of Nature, Science \& Technology, 2(2021), 7-11. https://doi.org/10.36937/janset.2021.002.002. 